\title{
The centrally restricted diffusion sign on MRI for assessment of radiation necrosis in metastases treated with stereotactic radiosurgery
}

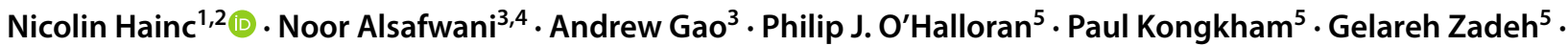 \\ Enrique Gutierrez ${ }^{6} \cdot$ David Shultz $^{6} \cdot$ Timo Krings $^{1,7} \cdot$ Paula Alcaide-Leon $^{1,7}$
}

Received: 22 September 2021 / Accepted: 16 October 2021 / Published online: 24 October 2021

(c) The Author(s) 2021

\begin{abstract}
Purpose Differentiation of radiation necrosis from tumor progression in brain metastases treated with stereotactic radiosurgery (SRS) is challenging. For this, we assessed the performance of the centrally restricted diffusion sign.

Methods Patients with brain metastases treated with SRS who underwent a subsequent intervention (biopsy/resection) for a ring-enhancing lesion on preoperative MRI between 2000 and 2020 were included. Excluded were lesions containing increased susceptibility limiting assessment of DWI. Two neuroradiologists classified the location of the diffusion restriction with respect to the post-contrast T1 images as centrally within the ring-enhancement (the centrally restricted diffusion sign), peripherally correlating to the rim of contrast enhancement, both locations, or none. Measures of diagnostic accuracy and 95\% CI were calculated for the centrally restricted diffusion sign. Cohen's kappa was calculated to identify the interobserver agreement.

Results Fifty-nine patients ( 36 female; mean age 59, range 40 to 80 ) were included, 36 with tumor progression and 23 with radiation necrosis based on histopathology. Primary tumors included 34 lung, 12 breast, 5 melanoma, 3 colorectal, 2 esophagus, 1 head and neck, 1 endometrium, and 1 thyroid. The centrally restricted diffusion sign was seen in 19/23 radiation necrosis cases (sensitivity 83\% (95\% CI 63 to 93\%), specificity 64\% (95\% CI 48 to 78\%), PPV 59\% (95\% CI 42 to $74 \%$ ), NPV 85\% (95\% CI 68 to 94\%)) and 13/36 tumor progression cases (difference p $<0.001$ ). Interobserver agreement was substantial, at 0.61 (95\% CI 0.45 to 70.8$)$.
\end{abstract}

Conclusion We found a low probability of radiation necrosis in the absence of the centrally restricted diffusion sign.

Keywords Diffusion magnetic resonance imaging $\cdot$ Radiation surgery $\cdot$ Radiation injuries $\cdot$ Necrosis

\begin{tabular}{|c|c|}
\hline \multicolumn{2}{|c|}{ Abbreviations } \\
\hline $\mathrm{ADC}$ & Apparent diffusion coefficient \\
\hline DCE & Dynamic contrast enhanced \\
\hline DSC & Dynamic susceptibility contrast \\
\hline DWI & Diffusion weighted imaging \\
\hline IR-FSPGR & $\begin{array}{l}\text { Volumetric T1-weighted fast spoiled grass } \\
\text { sequence with IR preparation }\end{array}$ \\
\hline MP-RAGE & $\begin{array}{l}\text { T1-weighted magnetization prepared rapid } \\
\text { gradient echo }\end{array}$ \\
\hline NPV & Negative predictive value \\
\hline PACS & Picture archiving and communication system \\
\hline PPV & Positive predictive value \\
\hline
\end{tabular}

Nicolin Hainc

Nicolin.hainc@usz.ch

Extended author information available on the last page of the article

\begin{tabular}{|c|c|}
\hline ROI & Region of interest \\
\hline STARD & $\begin{array}{l}\text { Standards for the Reporting of Diagnostic } \\
\text { Accuracy Studies }\end{array}$ \\
\hline SRS & Stereotactic radiosurgery \\
\hline SWI & Susceptibility weighted image \\
\hline WBRT & Whole brain radiotherapy \\
\hline
\end{tabular}

\section{Introduction}

Brain metastases are the most common form of intracranial tumor in the adult population and their incidence appears to be increasing due to longer patient survival and improving imaging techniques $[1,2]$. Stereotactic radiosurgery (SRS) is a treatment mainstay in patients with limited $(<5)$ metastases and is increasingly being used in patients with multiple $(\geq 5)$ metastases [3-5]. Assessing treatment response after 
SRS remains a challenge as a lesion size increase can be seen in up to $32 \%$ of radiated metastases, representing either radiation necrosis or true progression [6], and differentiation of these two entities is difficult based on conventional imaging alone [7].

Radiation necrosis can be characterized by a transient or progressive increase in enhancing lesion size due to inflammatory changes, and mimics tumor progression on imaging $[8,9]$. These lesions may stabilize or subside without additional therapy but imaging diagnosis usually requires several months of follow-up [10, 11]. In the case of progression, further treatment including surgical resection should be considered early for optimal local control [12]. While surgery is indicated in some cases of radiation necrosis, other cases in which it is mistaken for progression will result in unnecessary procedures or further radiation, which carry their own inherent risks, or suboptimal systemic therapies tailored to true progression [13]. Multiple advanced imaging techniques including dynamic contrast enhanced (DCE) and dynamic susceptibility contrast (DSC) MR-perfusion, MR-spectroscopy, intravoxel incoherent motion perfusion (IVIM) [14-22], and nuclear medicine studies including 18F-fluoro-ethyl-1-tyrosine positron emission tomography (18F-FET PET) [23] and 11C-methionine PET (11C METPET) [24] have been applied to differentiate radiation necrosis from tumor progression.

Diffusion weighted imaging (DWI) and the derived apparent diffusion coefficient (ADC) is a biomarker measuring water mobility in tissue providing indirect information on the tissue micro-environment [25-27]. Previous studies on brain metastases after SRS using ADC are based on quantitative differences on serial follow up imaging or require time-consuming lesion component segmentation [14, 25, $28,29]$. In a recent study on high-grade gliomas, Zakhari et al. [30] describe central diffusion restriction within a ring enhancing lesion to be indicative of radiation necrosis on visual analysis alone, with a study by Alcaide et al. validating this finding [31].

The aim of this study was to assess the diagnostic accuracy of the centrally restricted diffusion sign to differentiate radiation necrosis from tumor progression in patients with brain metastases treated with SRS, with ground truth determined by histopathology.

\section{Materials and methods}

\section{Subjects}

Institutional Review Board approval was obtained for this single center retrospective study. A Laboratory Information System search and Neurosurgery Archive search was performed using keywords "radiation", "treatment", and "metastasis" from 2000-2020. We included patients with SRS treated brain metastasis demonstrating a ring-enhancing lesion with central necrosis (on MRI) who subsequently underwent resection or biopsy. Excluded were patients without available pre-surgical/biopsy MRI studies in our picture archiving and communication system (PACS). Furthermore, patients lacking central necrosis on MRI were excluded. Central necrosis on MRI was defined as a nonenhancing region surrounded by contrast enhancement with the sum of biperpendicular diameters $>10 \mathrm{~mm}$. Finally, patients with increased susceptibility within the lesions were also excluded as assessment of DWI is limited in these cases [32].

\section{MR acquisition}

MR examinations were performed on $1.5 \mathrm{~T}$ and $3 \mathrm{~T}$ scanners (General Electric Healthcare, Waukesha, WI and Siemens Healthineers AG, Erlangen, Germany) using an eight-channel phased-array head coil. T1 weighted sequences were acquired before and after a bolus injection of $0.1 \mathrm{mmol} /$ $\mathrm{kg}$ body weight Gadobutrol (Gadovist, BayerHealthCare, Berlin, Germany). For T1 on GE, the volumetric T1-weighted fast spoiled grass sequence with IR preparation (IR-FSPGR) was acquired with following parameters: $\mathrm{TR} / \mathrm{TE}=7.22-9.00 / 1.48-4.20 \mathrm{~ms}$, matrix $=256 \times 224-256$, slice thickness $=1.0-2.0 \mathrm{~mm}$, FOV $=24-25 \times 24-25 \mathrm{~cm}$. For $\mathrm{T} 1$ on Siemens, the volumetric T1-weighted magnetization prepared rapid gradient echo (MP-RAGE) was acquired with following parameters: TR/TE $=2200.00 / 2.47 \mathrm{~ms}$, matrix $=256 \times 256$, slice thickness $=1.0 \mathrm{~mm}$, FOV $25 \times 25 \mathrm{~cm}$. DWI sequence parameters: TR/TE $=5600.00$ $9000.00 / 69.20-94.00 \mathrm{~ms}$, matrix $=128-256 \times 128-232$, slice thickness $=4.0-5.0 \mathrm{~mm}, \mathrm{FOV}=19.9-26 \mathrm{~cm} \times 22-26 \mathrm{~cm}$, $\mathrm{b}=1000 \mathrm{~s} / \mathrm{mm}^{2}$. ADC maps were generated from diffusion images. For GE, the $\mathrm{T} 2 *$-weighted sequence was acquired with following parameters: TR/TE $=4000.00 / 30.00 \mathrm{~ms}$, FOV $=22-24 \times 22-24$, matrix $=384 \times 256$, slice thickness $=5 \mathrm{~mm}$. For Siemens, the susceptibility weighted image (SWI) sequence was acquired with following parameters: $\mathrm{TR} / \mathrm{TE}=49.00 / 40.00$, matrix $=256 \times 177$, slice thickness $=2.0-2.6 \mathrm{~cm}, \mathrm{FOV}=20.1 \times 23.0 \mathrm{~cm}$.

\section{Image interpretation}

Screening of patients for inclusion in the study was performed by two neuroradiologists with 10 years and 7 years of experience (PA-L and NH). Patients demonstrating susceptibility artifact due to blood products within the treated lesion or lacking an area of necrosis were excluded. For the included patients, readers individually evaluated all cases for presence and location of the diffusion restriction using b1000 trace images and ADC maps in conjunction with post 
contrast T1 images in order to classify the lesions according to four different patterns. Disagreements were resolved by consensus. A hyperintensity on b1000 trace images with corresponding low ADC, lower than normal appearing white matter, was considered restricted diffusion. Diffusion restriction found within the boundaries of the ring enhancing lesion defined the centrally restricted diffusion sign. Four different diffusion patterns were thus possible and were categorized as follows: (1) "central" i.e. centrally within the ring enhancing lesion (the centrally restricted diffusion sign), (2) "peripheral" i.e. peripherally correlating only to the rim of contrast enhancement, (3) "both" i.e. diffusion restriction both centrally and peripherally (which thus includes central diffusion restriction and is counted as containing the centrally restricted diffusion sign), and (4) "no" i.e. no diffusion restriction.

True positives, true negatives etc. are defined based on the presence or absence of the centrally restricted diffusion sign on MRI with histopathology taken as the ground truth. Cases of histopathological radiation necrosis containing the centrally restricted diffusion sign on MRI, either through pattern 1 "central" or pattern 3 "both," are counted as true positive cases. Cases of histopathological tumor progression containing these patterns are counted as false positive cases. Conversely, cases of histopathological tumor progression not containing the centrally restricted diffusion sign on MRI, either through pattern 2 "peripheral" or pattern 4 "no" are counted as true negative cases. Cases of histopathological radiation necrosis containing these patterns are counted as false negative cases.

\section{Histopathology interpretation}

All specimens were fixed in formalin with routine preparation of hematoxylin and eosin-stained slides. Final diagnosis of radiation necrosis or tumor progression was made by expert pathologists based on the presence or absence of viable tumor with possible perivascular or intravascular distributions, necrotic tumor, fibrinoid necrosis, hemorrhage, hyalinization and thrombosis of the blood vessels, foamy macrophages, hemosiderin-laden macrophages, reactive gliosis, dystrophic calcification, and ghost cells, as was described in previous studies focusing on SRS of brain metastases [33, 34]. For subanalysis of all tumor progression cases, percentages of tumor cells for cases with and without the centrally restricted diffusion sign were recorded.

\section{Statistical analysis}

Interobserver agreement among the four different diffusion patterns was identified using Cohen's kappa [35]. A $\kappa$ value of 0.2 indicates slight agreement; $0.21-0.4$ fair agreement; $0.41-0.6$ moderate agreement; $0.61-0.80$ substantial agreement, and 0.81-1.00 almost perfect agreement [36]. Measures of diagnostic accuracy and 95\% CI were calculated for the centrally restricted diffusion sign indicating radiation necrosis. To investigate the performance of the centrally restricted diffusion sign to differentiate radiation necrosis from tumor progression, the two-sided Fisher's exact test was performed. To further assess the tumor progression group by comparing cases containing (i.e. false positives) and not containing (i.e. true negatives) the centrally restricted diffusion sign, the Mann-Whitney $U$ test was performed for variables including histopathologic tumor fraction, patient age, days from SRS to MRI, radiation dose, and prior WBRT through comparison of centrally restricted and non-centrally restricted tumor progression cases. Significance was set to $p<0.05$. Statistical analyses were performed using GraphPad Prism 8.2.1.

\section{Results}

\section{Patients}

One hundred forty one patients with brain metastases treated with SRS who underwent a subsequent intervention (biopsy/ resection) between February 2000 to February 2020 were retrospectively reviewed. After excluding patients without preoperative MRI in our PACS $(n=14)$, with extra-axial metastases $(n=2)$, lacking areas of necrosis $(n=17)$ or demonstrating susceptibility artefact limiting DWI assessment $(n=49)$, a total of 59 patients were included ( 36 female and 23 male) with a mean age of 59 years (range 40 to 80). Primary tumor histology was determined as follows: 34 lung, 12 breast, 5 melanoma, 3 colorectal, 2 esophagus, 1 head and neck, 1 endometrium, and 1 thyroid. Surgical resection was performed in 58 patients, while biopsy was performed in one patient. Mean time period from SRS to imaging was 403 days (SD 226, range 71 to 1001 days). Mean time period from SRS to surgery was 410 days (SD 223, range 71 to 1001 days). The date of SRS was available for 52/59 patients. Mean SRS dose was 18 Gy (SD 3, range 10 to $27 \mathrm{~Gy}$ ). Dose information was available for 44/59 patients. 18 patients had additional whole brain radiotherapy (WBRT) prior to SRS, 2 concomitant, and 5 following SRS. One patient had prior SRS to the same site. Thirteen patients underwent cavity SRS following initial surgical resection (4 with progressive tumor, 9 with radiation necrosis). The Standards for the Reporting of Diagnostic Accuracy Studies (STARD) flow diagram is shown in Fig. 1.

\section{Predictive value of the patterns}

Of the 59 included patients, 23 (39\%) were determined to have radiation necrosis by histopathology with the remaining 36 
Fig. 1 Standards for the reporting of diagnostic accuracy studies flow diagram. $R N$ radiation necrosis, $T P$ tumor progression

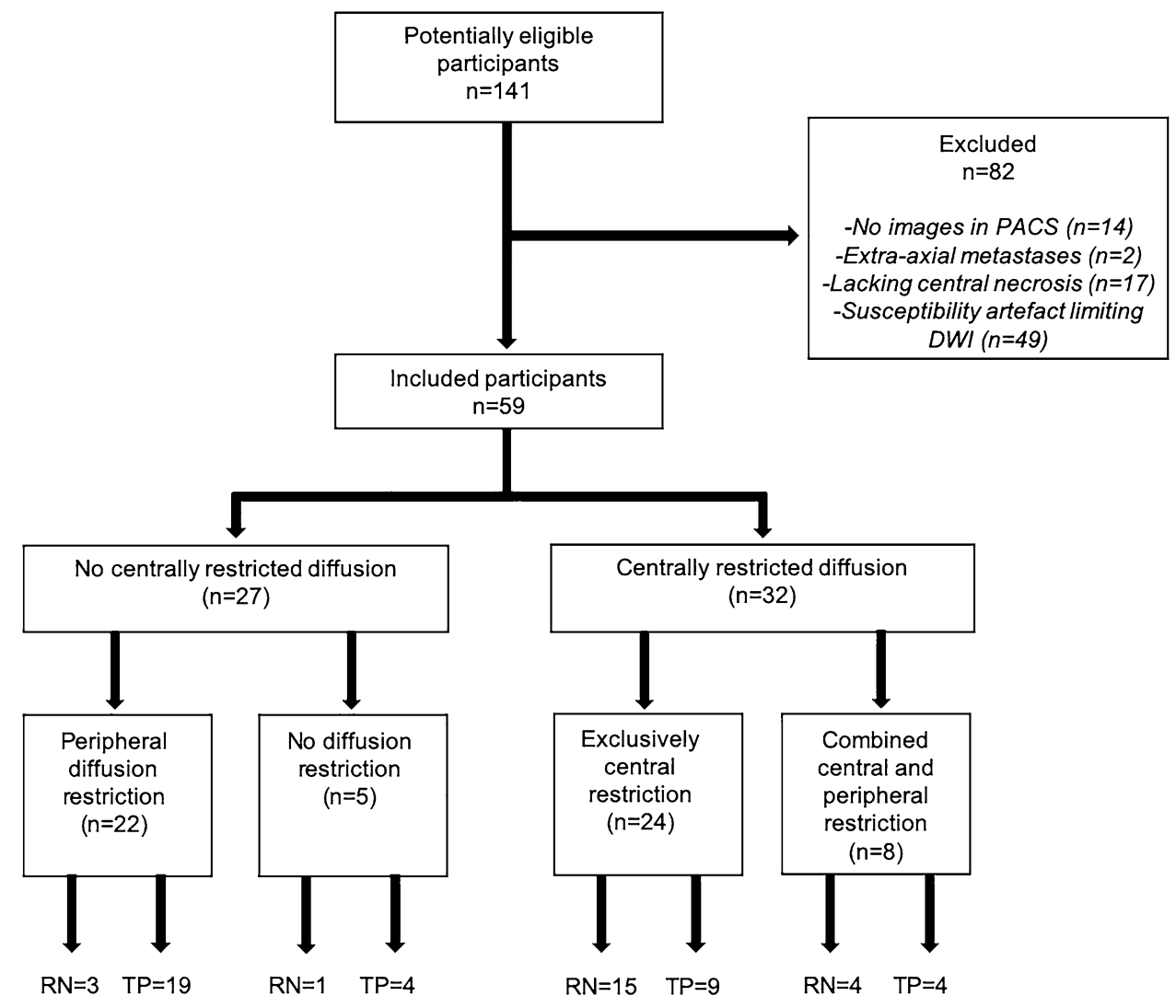

(61\%) determined to have tumor progression. Of the radiation necrosis cases, the centrally restricted diffusion sign was found in 19/23 (83\%) cases (Fig. 2). This included 15 cases with central diffusion restriction, i.e. exclusively within the ring enhancing lesion, and 4 with central diffusion restriction combined with peripheral diffusion restriction (i.e. overlapping the ring enhancement) (Table 1). The four cases not demonstrating either of these two patterns included one with no diffusion restriction and three with peripheral diffusion restriction only. Examples of the four diffusion patterns described are shown in Fig. 3.

For tumor progression cases, peripheral diffusion restriction was seen in 19/36 (53\%) cases and no diffusion restriction was seen in 4/36 (11\%) cases. The remaining $13(36 \%)$ cases were false positives containing the centrally restricted diffusion pattern ( 9 central, 4 central and peripheral).

The centrally restricted diffusion sign had a sensitivity of $83 \%$ (95\% CI 63 to $93 \%$ ) and a specificity of $64 \%$ (95\% CI 48 to $78 \%$ ) for radiation necrosis, with a positive predictive value (PPV) of 59\% (95\% CI 42 to $74 \%$ ), and a negative predictive value (NPV) of $85 \%$ (95\% CI 68 to 94\%). The presence of the centrally restricted diffusion sign significantly differentiated radiation necrosis from tumor progression $(\mathrm{p}<0.001)$.

\section{Interobserver agreement}

The $\kappa$ value was 0.61 (95\% CI 0.45 to 70.8 ) for the differentiation of the four diffusion patterns.

\section{Subanalysis of tumor progression cases}

The relatively high number of tumor progression cases with central diffusion restriction (13/36 (36\%), i.e. false positives) prompted further subanalysis. In a subgroup analysis differentiating among tumor progression cases with the centrally restricted diffusion sign (i.e. false positives; 7 lung, 4 breast, 1 melanoma, 1 head and neck) and without the centrally restricted diffusion sign (i.e. true negatives; 13 lung, 4 breast, 2 colorectum, 2 esophagus, 1 melanoma, 1 endometrium) there was no difference in histopathologic tumor fraction $(38 \pm 21 \%$ vs $46 \pm 23 \%)$, patient age (62 \pm 9 years vs $60 \pm 11$ years), days from SRS to MRI ( $392 \pm 221$ days vs $415 \pm 236$ days), radiation dose $(17.8 \pm 5.0$ Gy vs $17.8 \pm 2.7 \mathrm{~Gy})$, or prior WBRT ( $38 \%$ of cases vs $52 \%$ of cases) (Table 2 ). 

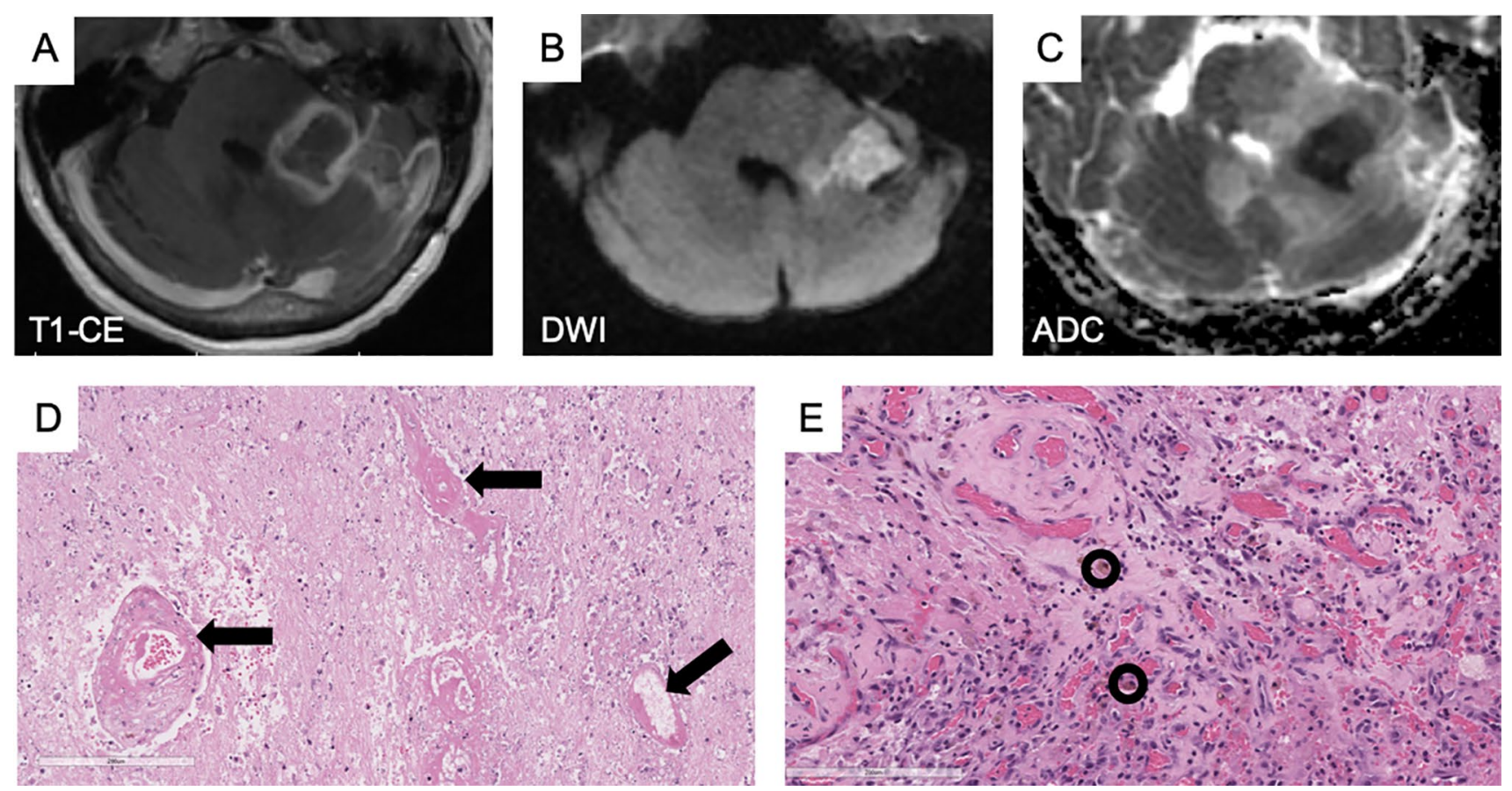

Fig. 2 Histopathologic correlation of a surgically resected lesion containing the centrally restricted diffusion sign, involving the left middle cerebellar peduncle, determined to represent radiation necrosis. A Contrast enhanced T1-weighted image demonstrating ring enhancement. B Diffusion weighted image and C ADC map demonstrating restricted diffusion within the ring enhancing lesion. Corresponding hematoxylin and eosin stained sections showing morphologic features of radiation effects composed of $\mathbf{D}$ coagulative-type necrosis and vasculopathic changes including angionecrosis, mural hyalinization, and luminal stenosis (black arrows). E There are aggregates of vascular proliferation with hemosiderin-laden macrophages (black circles)
Table 1 Number of subjects with radiation necrosis and tumor progression based on histopathology and the corresponding findings on MRI

\begin{tabular}{lll}
\hline Histopathology qround truth) & Central restriction (MRI) & No central restriction (MRI) \\
\hline Radiation necrosis & 19 True positive cases & 4 False negative cases \\
$\mathrm{n}=23$ & 15 "central" pattern & 3 "peripheral" pattern \\
& 4 "both" pattern & 1 "no" pattern \\
Tumor progression & 13 False positive cases & 23 True negative cases \\
$\mathrm{n}=36$ & 9 "central" pattern & 19 "peripheral" pattern \\
& 4 "both" pattern & 4 "no" pattern \\
\hline
\end{tabular}

True positives, false positives, true negatives, and false negatives are defined within the table with histopathology taken as the ground truth

\section{Discussion}

Our study demonstrates a low probability of radiation necrosis in SRS treated brain metastases in the absence of the central diffusion restriction sign, with a negative predictive value of $85.2 \%$, a sensitivity of $83 \%$, and a specificity of $64 \%$. This is comparable to advanced MR imaging techniques for differentiating radiation necrosis from tumor progression including DSC Perfusion (rCBV) (Hoefnagels et al. sensitivity 70\%, specificity 92.9\% [15], Barajas et al. sensitivity $91.3 \%$, specificity $72.7 \%$ [16]), IVIM (sensitivity $89.0 \%$, specificity $93.4 \%$ ) [21], and nuclear medicine studies including 11C MET-PET (sensitivity $82.0 \%$, specificity $75.0 \%$ ) [24]. Other nuclear medicine studies including 18F-FET PET appear to have the highest accuracy with a sensitivity of $95 \%$ and a specificity of $91 \%$ [23], increasing to $100 \%$ and $100 \%$, respectively, for glioma studies [37, 38]. Notably, not all cases within these studies had gold standard histopathological verification of radiation necrosis or tumor progression which may explain some discrepancies to our study [15, 16, 21, 23, 24, 38]. The substantial inter-reader agreement $(\mathrm{kappa}=0.61)$, lack of required region of interest (ROI) delineation, quantification, or incorporation of prior imaging studies for longitudinal assessment suggests the centrally restricted diffusion 
Fig. 3 The four diffusion patterns. 1A: a ring enhancing lesion (T1-CE) with central diffusion restriction (1B, DWI), "central" pattern, histologic radiation necrosis; $2 \mathrm{~A}$ : a ring enhancing lesion (T1-CE) with peripheral diffusion restriction (2B, DWI) overlapping the enhancing component, "peripheral" pattern, histologic tumor progression; $3 \mathrm{~A}$ a ring enhancing lesion (T1-CE) with central and peripheral diffusion restriction (3B, DWI), "both" pattern, histologic radiation necrosis; 4A: a ring enhancing lesion (T1-CE) without associated diffusion restriction (4B, DWI), "no" pattern, histologic tumor progression
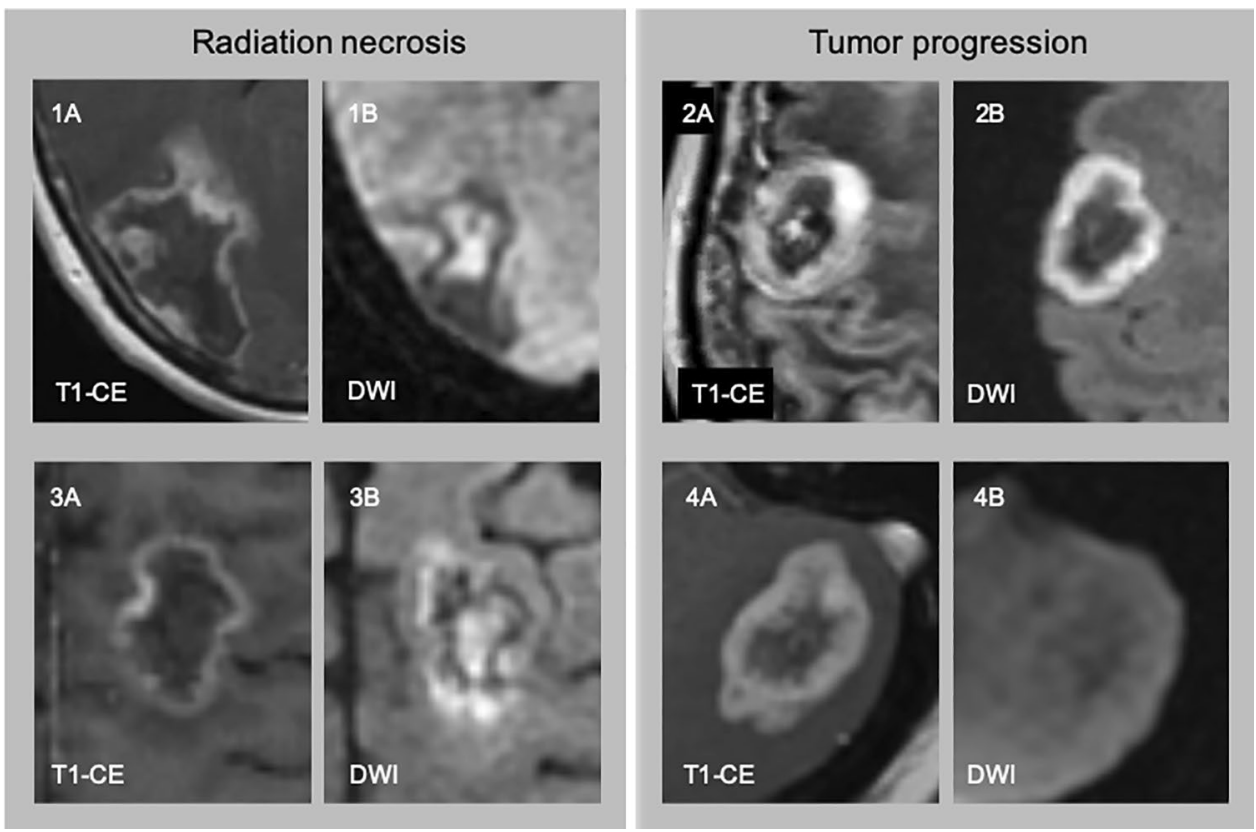

Table 2 Assessment of all Tumor Progression cases comparing false positives (central diffusion restriction) and true negatives (no central diffusion restriction)

\begin{tabular}{llll}
\hline $\begin{array}{l}\text { Tumor progression cases } \\
\mathrm{n}=36\end{array}$ & $\begin{array}{l}\text { Central restriction } \\
\mathrm{n}=13 \\
\text { (false positive cases) }\end{array}$ & $\begin{array}{l}\text { No central restriction } \\
\mathrm{n}=23 \\
\text { (true negative cases) }\end{array}$ & p value \\
\hline Histopathologic tumor Fraction (\%) & $38 \pm 21$ & $46 \pm 23$ & $>0.05$ \\
Patient age (years) & $62 \pm 9$ & $60 \pm 11$ & $>0.05$ \\
Time from SRS to MRI (days) & $392 \pm 221$ & $415 \pm 236$ & $>0.05$ \\
SRS dose (Gy) & $17.8 \pm 5.0$ & $17.8 \pm 2.7$ & $>0.05$ \\
Additional WBRT (n) & $5 / 13(38 \%)$ & $12 / 23(52 \%)$ & $>0.05$ \\
Primary tumor (n) & Lung (7) & Lung (13) & \\
& Breast (4) & Breast (4) & \\
& Melanoma (1) & Colorectum (2) & \\
& Head and neck (1) & Melanoma (2) & \\
& & Endometrium (1) & \\
\hline
\end{tabular}

$W B R T$ whole brain radiotherapy

No significant differences were found between the two groups sign to be a useful tool for the evaluation of radiation necrosis in clinical practice.

Our single timepoint location-based interpretation represents a paradigm shift in the application of DWI for postradiation brain metastases. Restricted diffusion (high DWI, low ADC) has often been used as a biomarker to represent hypercellularity and thus tumor, while increased or increasing diffusivity (low DWI, high ADC) was considered to represent necrosis, pseudoprogression, edema, or treatment response [14, 25, 28, 29]. Huang et al. presented a longitudinal imaging study on brain metastases treated with SRS through MRI studies performed at one week, one month, and three months post therapy to predict treatment success
[29]. Higher mean ADC values were reported in the group demonstrating radiation-induced central necrosis based on ROIs encompassing entire lesions. A longitudinal study by Chen et al. focusing on changes in $\mathrm{ADC}$ values described decreased or unchanging ADC indicative of non-response [28]. Knitter et al. evaluated the performance of DWI to differentiate true progression from pseudoprogression using pre- and post-treatment MRIs [14]. Here, ADC values between the two groups were not significantly different at any single timepoint, but an interval increase in ADC value was reported to identify pseudoprogression in $77 \%$ of lesions [14]. In the latter two studies [14, 28], DWI/ADC was assessed within the contrast enhancing regions only, 
with areas of necrosis excluded from the ROIs as they were not considered to contain relevant information, a methodology also seen in previous glioma studies [39, 40].

Shifting from a quantitative assessment to a qualitative, visual interpretation of DWI, Cha et al. differentiated radiation necrosis from tumor progression in SRS treated brain metastases using differing imaging patterns [20]. In their study, radiation necrosis was found only in their " 3 layer" pattern, with diffusion restriction located in the middle layer, corresponding to coagulative necrosis histologically. This methodology is similar to our study in that radiation necrosis was assessed for using visual analysis of imaging patterns alone without quantification or lesion segmentation. One main difference is that we did not differentiate layers within the contrast-enhancing rim, in line with previous glioma studies assessing for radiation necrosis using the centrally restricted diffusion sign [30,31]. The association between diffusion restriction and radiation necrosis has been shown in prior studies [20,30] and rat models [41] and appears to be due to coagulative necrosis with fibroblastic proliferation, hemosiderin laden macrophages, and inflammatory cells [34]. Comparisons have been drawn to a combination of a non-infectious pus-like material with abundant biological borders restricting diffusivity on a molecular scale [20].

For tumor progression, opposing findings have been described in the literature with regards to central diffusivity. While some previous studies associate absent diffusion restriction within the necrosis with tumor progression [30, $31,41]$, Cha et al. found all 5 cases of centrally restricted diffusion to represent tumor progression in their 16 subject cohort. Clearly, there is a discrepancy with regards to the diffusivity of the central necrosis in tumor progression cases which was also seen in our study, with central restriction seen in 13/36 (35\%) tumor progression cases and absent central restriction seen in the remaining 23/36 (65\%) tumor progression cases. Based on Cha et al.'s published data, we were not able to explain their discordant findings compared with our study [20]. On histopathological sub-analysis of our tumor progression cases, no differences were found between centrally restricting and centrally non-restricting cases with regards to histopathologic tumor fraction, patient age, time from SRS to MRI, or radiation dose. The true correlation between varying DWI signal characteristics in tumor progression is likely multifactorial and due in part to a combination of tumor characteristics, cell membrane density, macromolecular size and type, tissue oxygenation and viscosity, cell radiosensitivity, perfusion, and stage of evolution of necrosis [39, 42].

In this study, we only included histopathologically proven cases, which led to the small patient cohort. Histopathology did not differentiate lesion subsite based on MRI. Second, a large proportion of potential patients were excluded as they did not have ring-enhancing metastases or had increased susceptibility within the lesion, limiting the usefulness of the sign to a subset of patients. Finally, our cohort is heterogeneous with regards to treatment regimen and MR imaging technique, factors which are due to the retrospective nature of the study, however we feel this to be representative of what is seen on a daily basis.

To conclude, we found a low probability of radiation necrosis in the absence of the centrally restricted diffusion sign for brain metastases treated with SRS. The high negative predictive value of the centrally restricted diffusion sign combined with its substantial inter-reader agreement, lack of required region of interest delineation, quantification, or incorporation of prior imaging studies for longitudinal assessment suggests this to be a useful tool in clinical practice and adds to the growing body of evidence for differentiation of radiation necrosis from tumor progression. In future research, a multiparametric model incorporating the centrally restricted diffusion sign with advanced MR imaging techniques such as DSC Perfusion and IVIM may provide higher accuracies for the discrimination of radiation necrosis from tumor progression.

Acknowledgements The authors wish to acknowledge the generous support of the Slaight Family Foundation.

Author contributions All authors contributed to the study conception and design. Conceptualization was performed by NH and PA-L. Material preparation and data collection were performed by $\mathrm{NH}, \mathrm{NA}, \mathrm{AG}$, PJO, PK, GZ, EG, DS, TK, and PA-L. Analysis was performed by NH, NA, AG, and PA-L. The first draft of the manuscript was written by NH and all authors commented on previous versions of the manuscript. All authors read and approved the final manuscript.

Funding Open access funding provided by University of Zurich. None.

Data availability Study data is available upon reasonable request.

\section{Declarations}

Conflict of interest The authors declared that they have no conflict of interest.

Ethical approval Institutional Review Board approval was obtained for this single center retrospective study.

Consent to participate Waived by the IRB.

Consent for publication Waived by the IRB.

Open Access This article is licensed under a Creative Commons Attribution 4.0 International License, which permits use, sharing, adaptation, distribution and reproduction in any medium or format, as long as you give appropriate credit to the original author(s) and the source, provide a link to the Creative Commons licence, and indicate if changes were made. The images or other third party material in this article are included in the article's Creative Commons licence, unless indicated otherwise in a credit line to the material. If material is not included in the article's Creative Commons licence and your intended use is not 
permitted by statutory regulation or exceeds the permitted use, you will need to obtain permission directly from the copyright holder. To view a copy of this licence, visit http://creativecommons.org/licenses/by/4.0/.

\section{References}

1. Gavrilovic IT, Posner JB (2005) Brain metastases: epidemiology and pathophysiology. J Neurooncol 75:5-14. https://doi.org/10. 1007/s11060-004-8093-6

2. Sul J, Posner JB (2007) Brain metastases: epidemiology and pathophysiology. Cancer Treat Res 136:1-21. https://doi.org/10. 1007/978-0-387-69222-7_1

3. Tsao M, Xu W, Sahgal A (2012) A meta-analysis evaluating stereotactic radiosurgery, whole-brain radiotherapy, or both for patients presenting with a limited number of brain metastases. Cancer 118:2486-2493. https://doi.org/10.1002/cncr.26515

4. Yamamoto M, Serizawa T, Shuto T et al (2014) Stereotactic radiosurgery for patients with multiple brain metastases (JLGK0901): a multi-institutional prospective observational study. Lancet Oncol 15:387-395. https://doi.org/10.1016/S1470-2045(14)70061-0

5. Sahgal A, Ruschin M, Ma L et al (2017) Stereotactic radiosurgery alone for multiple brain metastases? A review of clinical and technical issues. Neuro-Oncol 19:ii2-ii15. https://doi.org/10.1093/ neuonc/nox001

6. Patel TR, McHugh BJ, Bi WL et al (2011) A comprehensive review of MR imaging changes following radiosurgery to 500 brain metastases. Am J Neuroradiol 32:1885-1892. https://doi. org/10.3174/ajnr.A2668

7. Lin NU, Lee EQ, Aoyama $\mathrm{H}$ et al (2015) Response assessment criteria for brain metastases: proposal from the RANO group. Lancet Oncol 16:e270-278. https://doi.org/10.1016/S1470-2045(15) 70057-4

8. Wong CS, Van der Kogel AJ (2004) Mechanisms of radiation injury to the central nervous system: implications for neuroprotection. Mol Interv 4:273-284. https://doi.org/10.1124/mi.4.5.7

9. Brandsma D, Stalpers L, Taal W et al (2008) Clinical features, mechanisms, and management of pseudoprogression in malignant gliomas. Lancet Oncol 9:453-461. https://doi.org/10.1016/S14702045(08)70125-6

10. Hygino da Cruz LC, Rodriguez I, Domingues RC et al (2011) Pseudoprogression and pseudoresponse: imaging challenges in the assessment of posttreatment glioma. AJNR Am J Neuroradiol 32:1978-1985. https://doi.org/10.3174/ajnr.A2397

11. Parvez K, Parvez A, Zadeh G (2014) The diagnosis and treatment of pseudoprogression, radiation necrosis and brain tumor recurrence. Int J Mol Sci 15:11832-11846. https://doi.org/10.3390/ ijms 150711832

12. Jeon YS, Koh Y-C, Song SW et al (2016) Palliative resection of metastatic brain tumors previously treated by stereotactic radiosurgery. Brain Tumor Res Treat 4:116-123

13. Ruzevick J, Kleinberg L, Rigamonti D (2014) Imaging changes following stereotactic radiosurgery for metastatic intracranial tumors: differentiating pseudoprogression from tumor progression and its effect on clinical practice. Neurosurg Rev 37:193-201. https://doi.org/10.1007/s10143-013-0504-8

14. Knitter JR, Erly WK, Stea BD et al (2018) Interval change in diffusion and perfusion MRI parameters for the assessment of pseudoprogression in cerebral metastases treated with stereotactic radiation. AJR Am J Roentgenol 211:168-175. https://doi.org/10. 2214/AJR.17.18890

15. Hoefnagels FWA, Lagerwaard FJ, Sanchez E et al (2009) Radiological progression of cerebral metastases after radiosurgery: assessment of perfusion MRI for differentiating between necrosis and recurrence. J Neurol 256:878-887. https://doi.org/10.1007/ s00415-009-5034-5

16. Barajas RF, Chang JS, Sneed PK et al (2009) Distinguishing recurrent intra-axial metastatic tumor from radiation necrosis following gamma knife radiosurgery using dynamic susceptibilityweighted contrast-enhanced perfusion MR imaging. AJNR Am J Neuroradiol 30:367-372. https://doi.org/10.3174/ajnr.A1362

17. Mitsuya K, Nakasu Y, Horiguchi S et al (2010) Perfusion weighted magnetic resonance imaging to distinguish the recurrence of metastatic brain tumors from radiation necrosis after stereotactic radiosurgery. J Neurooncol 99:81-88. https://doi.org/10.1007/ s11060-009-0106-z

18. Chernov MF, Hayashi M, Izawa M et al (2006) Multivoxel proton MRS for differentiation of radiation-induced necrosis and tumor recurrence after gamma knife radiosurgery for brain metastases. Brain Tumor Pathol 23:19-27. https://doi.org/10.1007/ s10014-006-0194-9

19. Rock JP, Hearshen D, Scarpace L et al (2002) Correlations between magnetic resonance spectroscopy and image-guided histopathology, with special attention to radiation necrosis. Neurosurgery 51:912-919; discussion 919-920. https://doi.org/10. 1097/00006123-200210000-00010

20. Cha J, Kim ST, Kim H-J et al (2013) Analysis of the layering pattern of the apparent diffusion coefficient (ADC) for differentiation of radiation necrosis from tumour progression. Eur Radiol 23:879-886. https://doi.org/10.1007/s00330-012-2638-4

21. Kim DY, Kim HS, Goh MJ et al (2014) Utility of intravoxel incoherent motion MR imaging for distinguishing recurrent metastatic tumor from treatment effect following gamma knife radiosurgery: initial experience. Am J Neuroradiol 35:2082-2090. https://doi. org/10.3174/ajnr.A3995

22. Detsky JS, Keith J, Conklin J et al (2017) Differentiating radiation necrosis from tumor progression in brain metastases treated with stereotactic radiotherapy: utility of intravoxel incoherent motion perfusion MRI and correlation with histopathology. J Neurooncol 134:433-441. https://doi.org/10.1007/s11060-017-2545-2

23. Galldiks N, Stoffels G, Filss CP et al (2012) Role of O-(2-(18) F-fluoroethyl)-L-tyrosine PET for differentiation of local recurrent brain metastasis from radiation necrosis. J Nucl Med Off Publ Soc Nucl Med 53:1367-1374. https://doi.org/10.2967/jnumed.112. 103325

24. Yomo S, Oguchi K (2017) Prospective study of 11C-methionine PET for distinguishing between recurrent brain metastases and radiation necrosis: limitations of diagnostic accuracy and longterm results of salvage treatment. BMC Cancer 17:713. https:// doi.org/10.1186/s12885-017-3702-X

25. Farjam R, Tsien CI, Feng FY et al (2014) Investigation of the diffusion abnormality index as a new imaging biomarker for early assessment of brain tumor response to radiation therapy. NeuroOncol 16:131-139. https://doi.org/10.1093/neuonc/not153

26. Patterson DM, Padhani AR, Collins DJ (2008) Technology insight: water diffusion MRI-a potential new biomarker of response to cancer therapy. Nat Clin Pract Oncol 5:220-233. https://doi.org/ $10.1038 /$ ncponc 1073

27. Padhani AR, Liu G, Mu-Koh D et al (2009) Diffusion-weighted magnetic resonance imaging as a cancer biomarker: consensus and recommendations. Neoplasia N Y N 11:102-125

28. Chen Z, Zu J, Li L et al (2017) Assessment of stereotactic radiosurgery treatment response for brain metastases using MRI based diffusion index. Eur J Radiol Open 4:84-88. https://doi.org/10. 1016/j.ejro.2017.06.002

29. Huang C-F, Chou H-H, Tu H-T et al (2008) Diffusion magnetic resonance imaging as an evaluation of the response of brain metastases treated by stereotactic radiosurgery. Surg Neurol 69:62-68; discussion 68. https://doi.org/10.1016/j.surneu.2007. 02.021 
30. Zakhari N, Taccone MS, Torres C et al (2018) Diagnostic Accuracy of Centrally Restricted Diffusion in the Differentiation of Treatment-Related Necrosis from Tumor Recurrence in HighGrade Gliomas. AJNR Am J Neuroradiol 39:260-264. https:// doi.org/10.3174/ajnr.A5485

31. Alcaide-Leon P, Cluceru J, Lupo JM et al (2020) Centrally reduced diffusion sign for differentiation between treatmentrelated lesions and glioma progression: a validation study. Am J Neuroradiol. https://doi.org/10.3174/ajnr.A6843

32. Atlas SW, Dubois P, Singer MB, Lu D (2000) Diffusion measurements in intracranial hematomas: implications for MR imaging of acute stroke. Am J Neuroradiol 21:1190-1194

33. Vellayappan B, Tan CL, Yong C et al (2018) Diagnosis and management of radiation necrosis in patients with brain metastases. Front Oncol 8:395. https://doi.org/10.3389/fonc.2018.00395

34. Jagannathan J, Bourne TD, Schlesinger D et al (2010) Clinical and pathological characteristics of brain metastasis resected after failed radiosurgery. Neurosurgery 66:208-217. https://doi.org/10. 1227/01.NEU.0000359318.90478.69

35. Cohen J (1960) A coefficient of agreement for nominal scales. Educ Psychol Meas 20:37-46. https://doi.org/10.1177/00131 6446002000104

36. Landis JR, Koch GG (1977) The measurement of observer agreement for categorical data. Biometrics 33:159-174

37. Verma N, Cowperthwaite MC, Burnett MG, Markey MK (2013) Differentiating tumor recurrence from treatment necrosis: a review of neuro-oncologic imaging strategies. Neuro-Oncol 15:515-534. https://doi.org/10.1093/neuonc/nos307

38. Pöpperl G, Götz C, Rachinger W et al (2004) Value of O-(2-[18F] fluoroethyl)-L-tyrosine PET for the diagnosis of recurrent glioma. Eur J Nucl Med Mol Imaging 31:1464-1470. https://doi.org/10. 1007/s00259-004-1590-1

39. Asao C, Korogi Y, Kitajima M et al (2005) Diffusion-weighted imaging of radiation-induced brain injury for differentiation from tumor recurrence. Am J Neuroradiol 26:1455-1460

40. Hein PA, Eskey CJ, Dunn JF, Hug EB (2004) Diffusion-weighted imaging in the follow-up of treated high-grade gliomas: tumor recurrence versus radiation injury. AJNR Am J Neuroradiol 25:201-209

41. Wang S, Chen Y, Lal B et al (2012) Evaluation of radiation necrosis and malignant glioma in rat models using diffusion tensor MR imaging. J Neurooncol 107:51-60. https://doi.org/10.1007/ s11060-011-0719-X

42. Schaefer PW, Grant PE, Gonzalez RG (2000) Diffusion-weighted MR imaging of the brain. Radiology 217:331-345. https://doi.org/ 10.1148/radiology.217.2.r00nv24331

Publisher's Note Springer Nature remains neutral with regard to jurisdictional claims in published maps and institutional affiliations.

\title{
Authors and Affiliations
}

\author{
Nicolin Hainc ${ }^{1,2}$. Noor Alsafwani ${ }^{3,4} \cdot$ Andrew Gao $^{3}$. Philip J. O'Halloran ${ }^{5} \cdot$ Paul Kongkham $^{5} \cdot$ Gelareh Zadeh $^{5}$. \\ Enrique Gutierrez ${ }^{6} \cdot$ David Shultz $^{6} \cdot$ Timo Krings $^{1,7} \cdot$ Paula Alcaide-Leon ${ }^{1,7}$ \\ Noor Alsafwani \\ Noor.Alsafwani@uhn.ca \\ Andrew Gao \\ Andrew.Gao@uhn.ca \\ Philip J. O'Halloran \\ philohalloran@rcsi.ie \\ Paul Kongkham \\ Paul.Kongkham@uhn.ca \\ Gelareh Zadeh \\ Gelareh.Zadeh@uhn.ca \\ Enrique Gutierrez \\ enrique.gutierrez@rmp.uhn.ca \\ David Shultz \\ david.shultz@rmp.uhn.ca \\ Timo Krings \\ 1 Department of Medical Imaging, University of Toronto, \\ Toronto, Canada \\ 2 Department of Neuroradiology, Clinical Neuroscience \\ Center, University Hospital Zurich, University of Zurich, \\ Frauenklinikstrasse 10, 8091 Zurich, Switzerland \\ 3 Laboratory Medicine Program, University Health Network, \\ Toronto, Canada \\ 4 Department of Pathology, College of Medicine, Imam \\ Abdulrahman Bin Faisal University (IAU), Dammam, \\ Saudi Arabia \\ 5 Neurosurgery, University Health Network, Toronto, Canada \\ 6 Radiation Oncology, Princess Margaret Cancer Centre, \\ Toronto, Canada \\ 7 Joint Department of Medical Imaging, University Health \\ Network, Toronto, Canada
}

Timo.Krings@uhn.ca

Paula Alcaide-Leon

Paulaalcaideleon@hotmail.com 